\title{
Characteristics and Maternal and Birth Outcomes of Hospitalized Pregnant Women with Laboratory-Confirmed COVID-19 - COVID-NET, 13 States, March 1-August 22, 2020
}

\begin{abstract}
Miranda J. Delahoy, $\mathrm{PhD}^{1,2}$; Michael Whitaker, MPH ${ }^{1,3}$; Alissa O’Halloran, MSPH ${ }^{1}$; Shua J. Chai, MD ${ }^{1,4}$; Pam Daily Kirley, MPH ${ }^{4}$; Nisha Alden, $\mathrm{MPH}^{5}$; Breanna Kawasaki, MPH${ }^{5}$; James Meek, MPH ${ }^{6}$; Kimberly Yousey-Hindes, MPH ${ }^{6}$; Evan J. Anderson, MD 7,8 ; Kyle P. Openo, DrPH ${ }^{7,8,9}$; Maya L. Monroe, MPH ${ }^{10}$; Patricia A. Ryan, MS ${ }^{10}$; Kimberly Fox, MPH ${ }^{11}$; Sue Kim, MPH ${ }^{11}$; Ruth Lynfield, MD ${ }^{12}$; Samantha Siebman, MPH ${ }^{12}$; Sarah Shrum Davis, MPH ${ }^{13}$; Daniel M. Sosin, MD ${ }^{14}$; Grant Barney, MPH ${ }^{15}$; Alison Muse, MPH ${ }^{15}$;

Nancy M. Bennett, MD ${ }^{16}$; Christina B. Felsen, MPH ${ }^{16}$; Laurie M. Billing, MPH ${ }^{17}$; Jessica Shiltz, MPH ${ }^{17}$; Melissa Sutton, MD ${ }^{18}$; Nicole West, MPH ${ }^{18}$; William Schaffner, $\mathrm{MD}^{19}$; H. Keipp Talbot, MD ${ }^{19}$; Andrea George, $\mathrm{MPH}^{20}$; Melanie Spencer, MPH ${ }^{20}$; Sascha Ellington, PhD ${ }^{1}$; Romeo R. Galang, MD ${ }^{1}$; Suzanne M. Gilboa, $\mathrm{PhD}^{1}$; Van T. Tong, MPH ${ }^{1}$; Alexandra Piasecki, MPH ${ }^{1,21}$; Lynnette Brammer, MPH ${ }^{1}$; Alicia M. Fry, MD ${ }^{1}$; Aron J. Hall, DVM ${ }^{1}$; Jonathan M. Wortham, MD ${ }^{1}$; Lindsay Kim, MD ${ }^{1}$; Shikha Garg, MD ${ }^{1}$; COVID-NET Surveillance Team
\end{abstract}

On September 16, 2020, this report was posted as an MMWR Early Release on the MMWR website (https://www.cdc.gov/mmwr).

Pregnant women might be at increased risk for severe coronavirus disease 2019 (COVID-19) $(1,2)$. The COVID-19-Associated Hospitalization Surveillance Network (COVID-NET) (3) collects data on hospitalized pregnant women with laboratory-confirmed SARS-CoV-2, the virus that causes COVID-19; to date, such data have been limited. During March 1-August 22, 2020, approximately one in four hospitalized women aged 15-49 years with COVID-19 was pregnant. Among 598 hospitalized pregnant women with COVID-19, 54.5\% were asymptomatic at admission. Among 272 pregnant women with COVID-19 who were symptomatic at hospital admission, $16.2 \%$ were admitted to an intensive care unit (ICU), and $8.5 \%$ required invasive mechanical ventilation. During COVID-19-associated hospitalizations, 448 of $458(97.8 \%)$ completed pregnancies resulted in a live birth and $10(2.2 \%)$ resulted in a pregnancy loss. Testing policies based on the presence of symptoms might miss COVID-19 infections during pregnancy. Surveillance of pregnant women with COVID-19, including those with asymptomatic infections, is important to understand the short- and long-term consequences of COVID-19 for mothers and newborns. Identifying COVID-19 in women during birth hospitalizations is important to guide preventive measures to protect pregnant women, parents, newborns, other patients, and hospital personnel. Pregnant women and health care providers should be made aware of the potential risks for severe COVID-19 illness, adverse pregnancy outcomes, and ways to prevent infection.

COVID-NET conducts population-based surveillance for laboratory-confirmed COVID-19-associated hospitalizations in 14 states encompassing 99 counties* (3). Thirteen states (California, Colorado, Connecticut, Georgia, Maryland, Michigan, Minnesota, New Mexico, New York, Ohio, Oregon, Tennessee, and Utah) contributed data to this report. Residents of the predefined surveillance catchment area who had a positive molecular test for SARS-CoV-2 during hospitalization or up to 14 days before hospital admission were classified as having a COVID-19-associated hospitalization and were included in COVID-NET surveillance. Persons included in COVID-NET surveillance are referred to as having COVID-19 throughout this report. SARS-CoV-2 testing was performed at the discretion of health care providers or through facility policies dictating uniform or criteria-based testing of patients upon admission. Trained surveillance officers performed medical chart abstractions for a convenience sample of hospitalizations using a standardized case report form. This analysis included women aged 15-49 years who were pregnant at hospital admission. Descriptive statistics were calculated for hospitalized pregnant women with complete

\footnotetext{
*Counties in COVID-NET surveillance: California (Alameda, Contra Costa, and San Francisco); Colorado (Adams, Arapahoe, Denver, Douglas, and Jefferson); Connecticut (Middlesex and New Haven); Georgia (Clayton, Cobb, DeKalb, Douglas, Fulton, Gwinnett, Newton, and Rockdale); Iowa (one county); Maryland (Allegany, Anne Arundel, Baltimore, Baltimore City, Calvert, Caroline, Carroll, Cecil, Charles, Dorchester, Frederick, Garrett, Harford, Howard, Kent, Montgomery, Prince George's, Queen Anne's, St. Mary's, Somerset, Talbot, Washington, Wicomico, and Worcester); Michigan (Clinton, Eaton, Genesee, Ingham, and Washtenaw); Minnesota (Anoka, Carver, Dakota, Hennepin, Ramsey, Scott, and Washington); New Mexico (Bernalillo, Chaves, Doña Ann, Grant, Luna, San Juan, and Santa Fe); New York (Albany, Columbia, Genesee, Greene, Livingston, Monroe, Montgomery, Ontario, Orleans, Rensselaer, Saratoga, Schenectady, Schoharie, Wayne, and Yates); Ohio (Delaware, Fairfield, Franklin, Hocking, Licking, Madison, Morrow, Perry, Pickaway, and Union); Oregon (Clackamas, Multnomah, and Washington); Tennessee (Cheatham, Davidson, Dickson, Robertson, Rutherford, Sumner, Williamson, and Wilson); and Utah (Salt Lake).
} 
chart review and discharge disposition (i.e., discharged or died during hospitalization). Women with one or more signs or symptoms included on the COVID-NET case report form (3) at the time of hospital admission were classified as symptomatic. Birth outcomes were described for pregnancies completed during a COVID-19-associated hospitalization. Reason for hospital admission was collected starting in June. Data were analyzed using SAS software (version 9.4; SAS Institute). This activity was reviewed by CDC and was conducted consistent with applicable federal law and CDC policy. ${ }^{\dagger}$ Sites obtained approval for COVID-NET

$\bar{\dagger} 45$ C.F.R. part 46.102(1)(2), 21 C.F.R. part 56; 42 U.S.C. Sect. 241 (d); 5 U.S.C. Sect. 552a; 44 U.S.C. Sect. 3501 et seq. surveillance from their state and local institutional review boards, as required.

During March 1-August 22, 2020, COVID-NET identified 7,895 hospitalized women aged 15-49 years with COVID-19; discharge disposition was determined, and chart review was completed for 2,318 (29.4\%) (Figure 1). Among 2,255 (97.3\%) women with information about pregnancy status, 598 (26.5\%) were pregnant, with median age 29 years. Among 577 (96.5\%) pregnant women with reported race and ethnicity, $42.5 \%$ were Hispanic or Latino (Hispanic), and 26.5\% were non-Hispanic Black (Black) (Table).

Among 596 women with COVID-19 whose pregnancy trimester was known, 14 (2.3\%), 61 (10.2\%), and 521 (87.4\%) were hospitalized during the first, second, and third trimesters,

FIGURE 1. Pregnancy status, signs and symptoms,* and birth outcomes ${ }^{\dagger, \S, \uparrow}$ among hospitalized women aged 15-49 years with COVID-19** COVID-NET, 13 states, ${ }^{\text {t† }}$ March 1-August 22, 2020

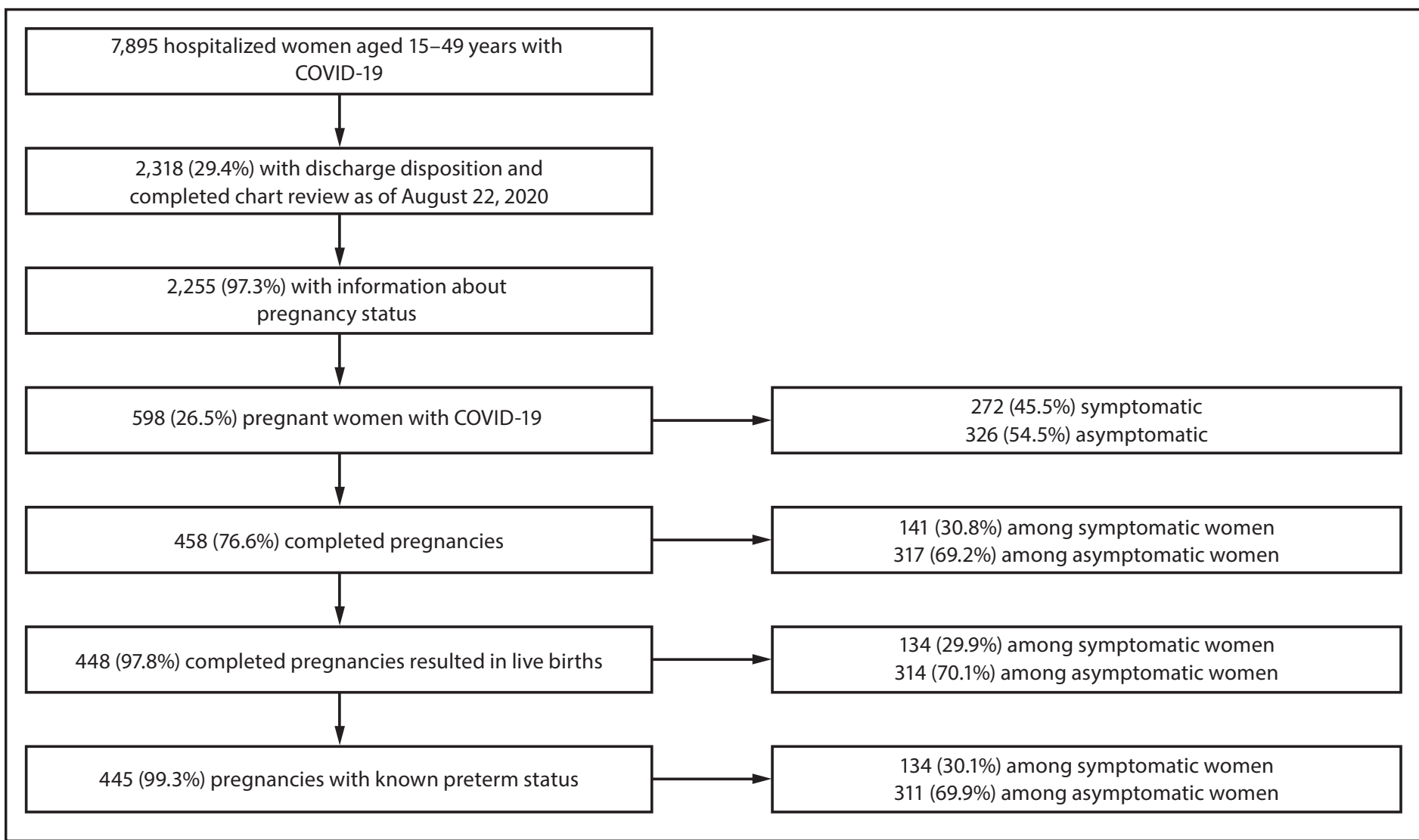

Abbreviations: COVID-19 = coronavirus disease 2019; COVID-NET = COVID-19-Associated Hospitalization Surveillance Network.

* Symptomatic women were those who had one or more signs or symptoms (fever/chills, cough, shortness of breath, muscle aches, nausea/vomiting, headache, sore throat, abdominal pain, chest pain, nasal congestion/rhinorrhea, decreased smell, decreased taste, diarrhea, upper respiratory illness/influenza-like illness, wheezing, hemoptysis/bloody sputum, conjunctivitis, rash, altered mental state, and seizure) at hospital admission; asymptomatic women did not have any of these signs or symptoms at admission.

† The 448 pregnancies resulting in live births resulted in the birth of 457 newborns; nine women had twins. Two newborns included in this category who were born alive subsequently died during the birth hospitalization.

$\S$ Ten completed pregnacies resulted in pregnancy losses. Pregnancy losses might include spontaneous abortion/miscarriage, therapeutic abortion, or stillbirth.

" Pregnancies with known preterm status were those resulting in a live birth for which the gestational age at delivery was known. For three pregnancies resulting in live births, the gestational age at the time of birth was unknown.

** Women residing in the predefined COVID-NET surveillance catchment with a positive real-time reverse transcription-polymerase chain reaction (RT-PCR) test for SARS-CoV-2, during hospitalization or up to 14 days before admission. Among the 597 (99.8\%) pregnant women for whom the COVID-19 test type was known, all had a positive RT-PCR test result; the COVID-19 test type for one pregnant woman with a positive COVID-19 test result was unknown.

${ }^{+\dagger}$ California, Colorado, Connecticut, Georgia, Maryland, Michigan, Minnesota, New Mexico, New York, Ohio, Oregon, Tennessee, and Utah. 
TABLE. Characteristics and outcomes of hospitalized pregnant women with COVID-19 - COVID-NET, 13 states, ${ }^{*}$ March 1-August 22, 2020

no./No. (\%)

\begin{tabular}{|c|c|c|c|}
\hline Characteristic & $\begin{array}{l}\text { Overall } \\
(\mathrm{N}=598)\end{array}$ & $\begin{array}{l}\text { Symptomatic at admission } \\
\qquad(n=272)\end{array}$ & $\begin{array}{l}\text { Asymptomatic at admission } \\
(\mathrm{n}=326)\end{array}$ \\
\hline \multicolumn{4}{|l|}{ Age group, yrs } \\
\hline $15-24$ & 167/598 (27.9) & $69 / 272(25.4)$ & $98 / 326(30.1)$ \\
\hline $25-34$ & 318/598 (53.2) & $143 / 272(52.6)$ & $175 / 326(53.7)$ \\
\hline $35-49$ & $113 / 598(18.9)$ & $60 / 272(22.1)$ & $53 / 326(16.3)$ \\
\hline \multicolumn{4}{|l|}{ Race/Ethnicity $(n=577)$} \\
\hline Hispanic or Latino & $245 / 577(42.5)$ & $131 / 265(49.4)$ & 114/312 (36.5) \\
\hline American Indian or Alaska Native, non-Hispanic & $8 / 577(1.4)$ & $4 / 265(1.5)$ & $4 / 312(1.3)$ \\
\hline Asian or Pacific Islander, non-Hispanic & $72 / 577(12.5)$ & $37 / 265(14.0)$ & $35 / 312(11.2)$ \\
\hline Black, non-Hispanic & $153 / 577(26.5)$ & $57 / 265(21.5)$ & $96 / 312(30.8)$ \\
\hline White, non-Hispanic & $97 / 577(16.8)$ & $35 / 265(13.2)$ & $62 / 312(19.9)$ \\
\hline Multiracial & $2 / 577(0.3)$ & $1 / 265(0.4)$ & $1 / 312(0.3)$ \\
\hline \multicolumn{4}{|l|}{ Pregnancy trimester at hospital admission $(n=596)$} \\
\hline First & $14 / 596(2.3)$ & 13/271 (4.8) & $1 / 325(0.3)$ \\
\hline Second & $61 / 596(10.2)$ & $50 / 271(18.5)$ & $11 / 325(3.4)$ \\
\hline Third & $521 / 596(87.4)$ & $208 / 271(76.8)$ & $313 / 325(96.3)$ \\
\hline \multicolumn{4}{|l|}{ Reason for hospital admission $(n=324)^{\dagger}$} \\
\hline COVID-19-related illness & $61 / 324(18.8)$ & $59 / 122(48.4)$ & $2 / 202(1.0)$ \\
\hline Obstetrics/Labor and delivery & $242 / 324(74.7)$ & $55 / 122(45.1)$ & $187 / 202(92.6)$ \\
\hline Other & $21 / 324(6.5)$ & $8 / 122(6.6)$ & $13 / 202(6.4)$ \\
\hline \multicolumn{4}{|l|}{ Underlying conditions } \\
\hline Any underlying condition or conditions & 123/598 (20.6) & $63 / 272(23.2)$ & $60 / 326(18.4)$ \\
\hline Asthma & 49/598 (8.2) & $30 / 272(11.0)$ & 19/326 (5.8) \\
\hline Cardiovascular disease (excludes hypertension) & $6 / 598(1.0)$ & $6 / 272(2.2)$ & $0 / 326(-)$ \\
\hline Chronic lung disease & $6 / 598(1.0)$ & $6 / 272(2.2)$ & $0 / 326(-)$ \\
\hline Chronic metabolic disease & $44 / 598(7.4)$ & $23 / 272(8.5)$ & $21 / 326(6.4)$ \\
\hline Diabetes mellitus ${ }^{\S}$ & $23 / 598(3.8)$ & $15 / 272(5.5)$ & $8 / 326(2.5)$ \\
\hline Thyroid dysfunction & $21 / 598(3.5)$ & 9/272 (3.3) & $12 / 326(3.7)$ \\
\hline Hypertension & $26 / 598(4.3)$ & $12 / 272(4.4)$ & $14 / 326(4.3)$ \\
\hline Liver disease & 10/598 (1.7) & $5 / 272(1.8)$ & $5 / 326(1.5)$ \\
\hline Neurologic conditions & $12 / 598(2.0)$ & $6 / 272(2.2)$ & $6 / 326(1.8)$ \\
\hline Other underlying condition or conditions ${ }^{\natural}$ & $7 / 598(1.2)$ & $3 / 272(1.1)$ & $4 / 326(1.2)$ \\
\hline \multicolumn{4}{|l|}{ Smoking } \\
\hline Current smoker & $13 / 598(2.2)$ & $8 / 272(2.9)$ & $5 / 326(1.5)$ \\
\hline Former smoker & $41 / 598(6.9)$ & 20/272 (7.4) & $21 / 326(6.4)$ \\
\hline Not a smoker/Unknown smoking history & $544 / 598(91.0)$ & 244/272 (89.7) & $300 / 326(92.0)$ \\
\hline \multicolumn{4}{|l|}{ Chest radiograph findings $(n=132)^{* *}$} \\
\hline Infiltrate/Consolidation & 103/132 (78.0) & $99 / 121(81.8)$ & $4 / 11(36.4)$ \\
\hline Bronchopneumonia/Pneumonia & $39 / 132(29.5)$ & $39 / 121(32.2)$ & $0 / 11(-)$ \\
\hline Pleural effusion & $2 / 132(1.5)$ & $1 / 121(0.8)$ & $1 / 11(9.1)$ \\
\hline \multicolumn{4}{|l|}{ Chest CT findings $(n=48)^{\dagger \dagger}$} \\
\hline Ground glass opacities & $21 / 48(43.8)$ & $17 / 40(42.5)$ & $4 / 8(50.0)$ \\
\hline Infiltrate/Consolidation & $31 / 48(64.6)$ & $28 / 40(70.0)$ & $3 / 8(37.5)$ \\
\hline Bronchopneumonia/pneumonia & $17 / 48(35.4)$ & $15 / 40(37.5)$ & $2 / 8(25.0)$ \\
\hline Pleural effusion & $7 / 48(14.6)$ & $5 / 40(12.5)$ & $2 / 8(25.0)$ \\
\hline \multicolumn{4}{|l|}{ COVID-19 investigational treatments } \\
\hline Received treatment (not mutually exclusive) & $52 / 598(8.7)$ & $43 / 272(15.8)$ & $9 / 326(2.8)$ \\
\hline Remdesivir & $18 / 598(3.0)$ & $18 / 272(6.6)$ & $0 / 326(-)$ \\
\hline Azithromycin $§ \S$ & $25 / 598(4.2)$ & $24 / 272(8.9)$ & $1 / 326(0.3)$ \\
\hline Hydroxychloroquine & $21 / 598(3.5)$ & $19 / 272(7.0)$ & $2 / 326(0.6)$ \\
\hline Convalescent plasma & $9 / 598(1.5)$ & 9/272 (3.3) & $0 / 326(0)$ \\
\hline Chloroquine & $1 / 598(0.2)$ & $1 / 272(0.4)$ & $0 / 326(0)$ \\
\hline Other & $17 / 598(2.8)$ & $10 / 272(3.7)$ & $7 / 326(2.2)$ \\
\hline Hospital length of stay, median (IQR), days & $2(2-3)$ & $3(2-5)$ & $2(2-3)$ \\
\hline ICU admission & $44 / 598(7.4)$ & $44 / 272(16.2)$ & $0 / 326(-)$ \\
\hline ICU length of stay, median (IQR), days $(n=41)^{\text {กๆ }}$ & $5(2-13)$ & $5(2-13)$ & - \\
\hline
\end{tabular}

See table footnotes on the next page. 
TABLE. (Continued) Characteristics and outcomes of hospitalized pregnant women with COVID-19 - COVID-NET, 13 states, ${ }^{*}$ March $1-$ August 22, 2020

\begin{tabular}{|c|c|c|c|}
\hline \multirow[b]{2}{*}{ Characteristic } & \multicolumn{3}{|c|}{ no./No. (\%) } \\
\hline & $\begin{array}{l}\text { Overall } \\
(\mathrm{N}=598)\end{array}$ & $\begin{array}{l}\text { Symptomatic at admission } \\
\qquad(n=272)\end{array}$ & $\begin{array}{l}\text { Asymptomatic at admission } \\
\qquad(\mathrm{n}=326)\end{array}$ \\
\hline \multicolumn{4}{|l|}{ Interventions } \\
\hline Invasive mechanical ventilation*** & $23 / 598(3.8)$ & $23 / 272(8.5)$ & $0 / 326(-)$ \\
\hline BIPAP/CPAP*** & $3 / 598(0.5)$ & $3 / 272(1.1)$ & $0 / 326(-)$ \\
\hline High flow nasal cannula*** & $5 / 598(0.8)$ & $5 / 272(1.8)$ & $0 / 326(-)$ \\
\hline Systemic steroids & $34 / 598(5.7)$ & $22 / 272(8.1)$ & $12 / 326(3.7)$ \\
\hline Vasopressors & $32 / 598(5.4)$ & $22 / 272(8.1)$ & 10/326 (3.1) \\
\hline ECMO & $2 / 598(0.3)$ & $2 / 272(0.7)$ & $0 / 326(-)$ \\
\hline Renal replacement therapy or dialysis & $2 / 598(0.3)$ & $2 / 272(0.7)$ & $0 / 326(-)$ \\
\hline \multicolumn{4}{|l|}{ New clinical discharge diagnoses $(n=554)^{t+\dagger}$} \\
\hline Acute respiratory distress syndrome & $15 / 554(2.7)$ & 14/251 (5.6) & $1 / 303(0.3)$ \\
\hline Acute respiratory failure & $41 / 554(7.4)$ & $41 / 251(16.3)$ & 0/303 (一) \\
\hline Pneumonia & $75 / 554(13.5)$ & $73 / 251(29.1)$ & $2 / 303(0.7)$ \\
\hline Sepsis & $21 / 554(3.8)$ & $21 / 251(8.4)$ & $0 / 303(-)$ \\
\hline In-hospital death & $2 / 598(0.3)$ & $2 / 272(0.7)$ & $0 / 326(-)$ \\
\hline \multicolumn{4}{|l|}{ Current pregnancy plurality } \\
\hline Singleton pregnancy & $567 / 598(94.8)$ & $253 / 272(93.0)$ & $314 / 326(96.3)$ \\
\hline Multiple pregnancy & $14 / 598(2.3)$ & $8 / 272(2.9)$ & $6 / 326(1.8)$ \\
\hline Unknown & $17 / 598(2.8)$ & $11 / 272(4.0)$ & $6 / 326(1.8)$ \\
\hline \multicolumn{4}{|l|}{ Pregnancy-associated conditions $(n=581)^{\S \S \S}$} \\
\hline Gestational diabetes & $64 / 581(11.0)$ & $31 / 261$ (11.9) & $33 / 320(10.3)$ \\
\hline Hypertensive disorders of pregnancy & $70 / 581(12.0)$ & $33 / 261(12.6)$ & $37 / 320(11.6)$ \\
\hline Intrauterine growth restriction & $11 / 581(1.9)$ & $4 / 261(1.5)$ & $7 / 320(2.2)$ \\
\hline None & $453 / 581(78.0)$ & $202 / 261(77.4)$ & $251 / 320(78.4)$ \\
\hline \multicolumn{4}{|l|}{ Pregnancy status at discharge or death } \\
\hline Still pregnant & $139 / 598(23.2)$ & $130 / 272(47.8)$ & $9 / 326(2.8)$ \\
\hline No longer pregnant & $458 / 598(76.6)$ & $141 / 272(51.8)$ & $317 / 326(97.2)$ \\
\hline Unknown & $1 / 598(0.2)$ & $1 / 272(0.4)$ & $0 / 326(-)$ \\
\hline \multicolumn{4}{|l|}{ Pregnancy outcomes $(n=458)$} \\
\hline Live birth**** & $448 / 458(97.8)$ & $134 / 141(95.0)$ & $314 / 317(99.1)$ \\
\hline Term live birth $(\geq 37 \mathrm{wks})^{+t+\dagger}$ & $389 / 445(87.4)$ & 103/134 (76.9) & 286/311 (92.0) \\
\hline Pre-term live birth $(<37 \mathrm{wks})^{++\dagger+}$ & $56 / 445(12.6)$ & $31 / 134(23.1)$ & 25/311 (8.0) \\
\hline Pregnancy loss $§ \S \S$ & $10 / 458(2.2)$ & $7 / 141(5.0)$ & $3 / 317(0.9)$ \\
\hline Pregnancy loss at $<20$ wks' gestation & $4 / 458(0.9)$ & $3 / 141(2.1)$ & $1 / 317(0.3)$ \\
\hline Pregnancy loss at $\geq 20 \mathrm{wks}^{\prime}$ gestation & $5 / 458(1.1)$ & $4 / 141(2.8)$ & $1 / 317(0.3)$ \\
\hline Pregnancy loss at unknown gestational age & $1 / 458(0.2)$ & $0 / 141(-)$ & $1 / 317(0.3)$ \\
\hline In-hospital newborn death ๆางๆา & $2 / 448(0.4)$ & $2 / 134(1.5)$ & $0 / 314(-)$ \\
\hline \multicolumn{4}{|l|}{ Mode of delivery $(n=458)$} \\
\hline Vaginal & $302 / 458(65.9)$ & $79 / 141(56.0)$ & $223 / 317(70.3)$ \\
\hline Cesarean section & $151 / 458(33.0)$ & $59 / 141(41.8)$ & $92 / 317(29.0)$ \\
\hline Unknown & $5 / 458(1.1)$ & $3 / 141(2.1)$ & $2 / 317(0.6)$ \\
\hline
\end{tabular}

Abbreviations: BIPAP/CPAP = bilevel positive airway pressure/continuous positive airway pressure; COVID-19= coronavirus disease 2019; $C T=$ computed tomography;

$\mathrm{ECMO}=$ extracorporeal membrane oxygenation; ICU = intensive care unit; IQR = interquartile range.

* California, Colorado, Connecticut, Georgia, Maryland, Michigan, Minnesota, New Mexico, New York, Ohio, Oregon, Tennessee, and Utah.

$\dagger$ Information not available for those hospitalized before June 2020.

$\S$ Does not include gestational diabetes.

" One or more other underlying conditions, which included blood disorders/hemoglobinopathy (four), immunocompromised condition (two), renal disease (one), and rheumatologic/autoimmune/inflammatory condition (one).

** Among those who had a chest radiograph performed during hospitalization or $\leq 3$ days before the hospital admission.

${ }^{+\dagger}$ Among those who had a chest CT/MRI performed during hospitalization or $\leq 3$ days before the hospital admission.

$\S \S$ If administered with another COVID-19 investigational treatment.

११ Includes women admitted to an ICU with a known ICU length of stay.

*** Highest level of respiratory support for each woman who needed respiratory support.

$\mathrm{tt \dagger}^{\mathrm{t}}$ Based on discharge summary diagnoses, for those who had discharge summaries.

$\S \S$ Among those with information on pregnancy-associated conditions.

ๆ१ศ Preeclampsia or gestational hypertension.

**** Number of pregnancies resulting in live birth; might have been a singleton or multiple delivery.

${ }^{+t+t}$ Among live births with known gestational age at delivery.

$\S \S \S$ Pregnancy losses might include spontaneous abortion/miscarriage, therapeutic abortion, or stillbirth.

ๆาศๆ The denominator refers to the 448 pregnancies resulting in live births. These 448 pregnancies resulted in the birth of 457 newborns; nine women had twins. The deaths of two newborns that occurred during the birth hospitalization were indicated on their mothers' hospital charts. 
respectively. The reason for hospital admission was reported for 324 women: $242(74.7 \%)$ were hospitalized for obstetric indications (including labor and delivery), 61 (18.8\%) for COVID-19-related illness, and 21 (6.5\%) for other reasons. The most common reason for admission during the first or second pregnancy trimester was COVID-19-related illness $(56.8 \%)$ and during the third trimester, obstetric indications $(81.9 \%)$. Among hospitalized pregnant women with COVID-19, 20.6\% had at least one underlying medical condition; asthma $(8.2 \%)$ and hypertension $(4.3 \%)$ were the most prevalent.

Overall, 272 (45.5\%) pregnant women with COVID-19 were symptomatic at the time of hospital admission, and 326 $(54.5 \%)$ were asymptomatic. Women hospitalized during the first or second trimester were more frequently symptomatic $(84.0 \%)$ than were those hospitalized during the third trimester (39.9\%). Among symptomatic women, the most commonly reported symptoms were fever or chills (59.6\%) and cough (59.2\%) (Figure 2).

Among 272 hospitalized symptomatic pregnant women, 44 $(16.2 \%)$ were admitted to an ICU and $23(8.5 \%)$ required invasive mechanical ventilation. Two $(0.7 \%)$ deaths were reported among symptomatic women. No asymptomatic women were admitted to an ICU, required invasive mechanical ventilation, or died.

At hospital discharge, 458 women (76.6\%) with COVID-19 had completed pregnancies, including $448(97.8 \%)$ that resulted in live births and $10(2.2 \%)$ in pregnancy losses (Figure 1). Pregnancy losses occurred among both symptomatic and asymptomatic hospitalized women with COVID-19 (Table). Four pregnancy losses $(0.9 \%$ of completed pregnancies) occurred at $<20$ weeks' gestation, five ( $1.1 \%)$ at $\geq 20$ weeks' gestation, and one $(0.2 \%)$ at unknown gestational age. Among 445 pregnancies resulting in live births with known gestational age at delivery, $87.4 \%$ were term births ( $\geq 37$ weeks' gestation), and $12.6 \%$ were preterm ( $<37$ weeks). Among pregnancies resulting in live births, preterm delivery was reported for $23.1 \%$ of symptomatic women and $8.0 \%$ of asymptomatic women. Two live-born newborns died during the birth hospitalization (Table); both were born to symptomatic women who required invasive mechanical ventilation.

\section{Discussion}

One in four women aged 15-49 years who had a COVID-19-associated hospitalization during March 1August 22, 2020 was pregnant, based on a convenience sample from COVID-NET. Approximately one half of pregnant women

FIGURE 2. Signs and symptoms* at hospital admission among symptomatic hospitalized pregnant women with COVID-19, ${ }^{\dagger}$ by pregnancy trimester — COVID-NET, 13 states, $\$$ March 1-August 22, 2020

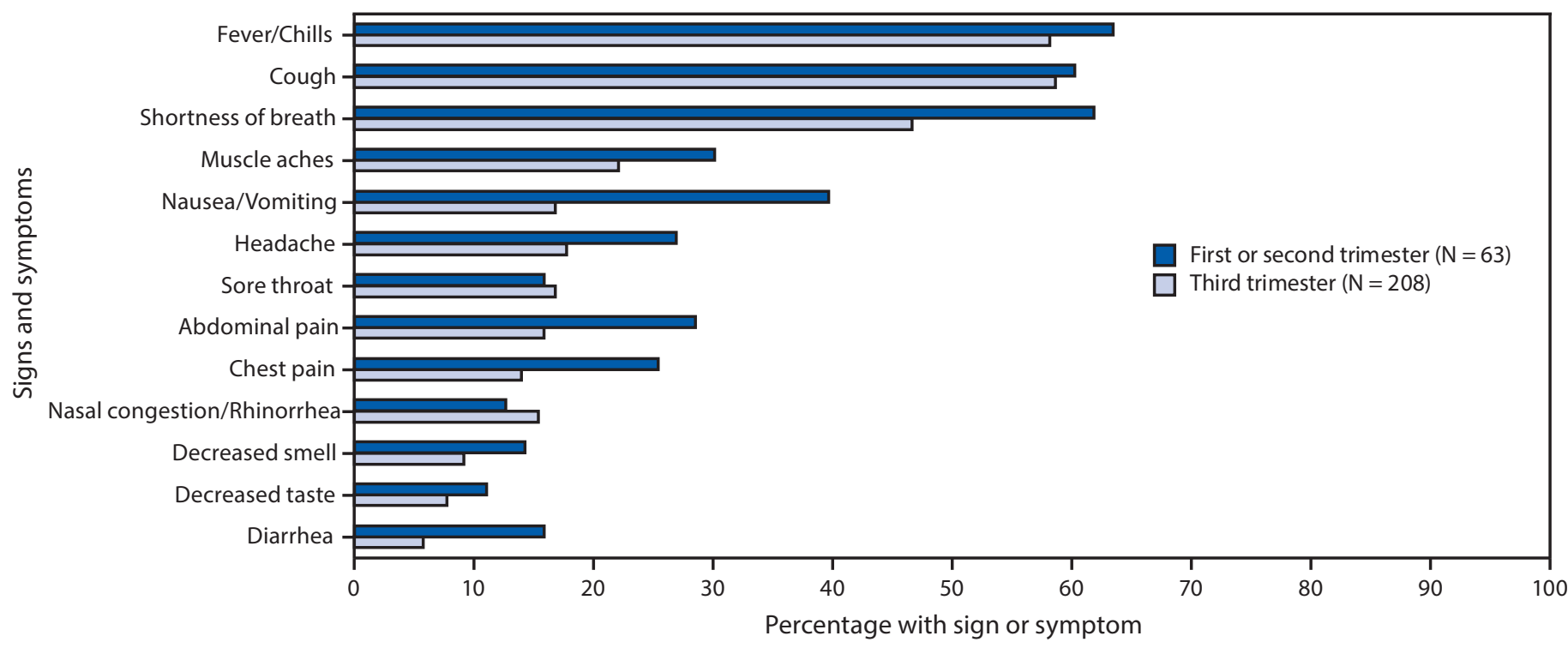

Abbreviations: COVID-19 = coronavirus disease 2019; COVID-NET = COVID-19-Associated Hospitalization Surveillance Network.

* Other signs and symptoms reported on the case report form were upper-respiratory illness/influenza-like illness (11 persons), wheezing (six), hemoptysis/bloody sputum (one), conjunctivitis (one), rash (one), altered mental state (one) and seizure (none). The symptoms decreased smell and decreased taste might not have been ascertained for cases admitted before April 1, 2020, when these symptoms were added as options on the case report form.

${ }^{\dagger}$ A total of 272 pregnant women with COVID-19 with at least one sign or symptom at the time of hospitalization were identified in COVID-NET. One hospitalized pregnant woman who was symptomatic at admission was not included in this figure because of missing pregnancy trimester.

$\S$ California, Colorado, Connecticut, Georgia, Maryland, Michigan, Minnesota, New Mexico, New York, Ohio, Oregon, Tennessee, and Utah. 
were asymptomatic at hospital admission. Among symptomatic pregnant women, $16.2 \%$ were admitted to an ICU, 8.5\% required invasive mechanical ventilation, and two died during COVID-19-associated hospitalizations; none of these outcomes occurred among asymptomatic pregnant women. Among all pregnancies completed during a COVID-19-associated hospitalization, 2.2\% resulted in pregnancy losses. Pregnancy losses occurred among both symptomatic and asymptomatic hospitalized women with COVID-19.

Approximately 5\% of women of reproductive age in the general population are pregnant at any given time (1). The proportion of hospitalized women aged 15-49 years with COVID-19 who were pregnant in this study (26.5\%) suggests that pregnant women have disproportionately higher rates of COVID-19-associated hospitalizations compared to nonpregnant women. Although COVID-19 might be more severe in pregnant women, other factors might also explain these higher hospitalization rates. Providers might have a lower threshold for admitting pregnant women for any reason. Some pregnant women with COVID-19 might be admitted solely to give birth. Pregnant women might also have a higher likelihood of being tested for COVID-19 upon admission than do nonpregnant women. Nevertheless, pregnant women account for a substantial proportion of COVID-19-associated hospitalizations among women of reproductive age.

The proportions of hospitalized pregnant women who were Hispanic (42.5\%) and Black (26.5\%) were higher than the overall proportions of women aged 15-49 years in the COVID-NET catchment area who were Hispanic (15.3\%) or Black (19.5\%). ${ }^{\S}$ Although the racial and ethnic composition of pregnant women in the catchment area is unknown, this report and an earlier study (1) suggest that pregnant women who are Hispanic or Black might have disproportionately higher rates of COVID-19-associated hospitalization, compared with those of pregnant women of other races and ethnicities. Long standing inequities in the social determinants of health, such as occupation and housing circumstances that make physical distancing challenging, have put some racial and ethnic minority groups at increased risk for COVID-19-associated illness and death $(4,5)$. Better understanding of the circumstances under which Hispanic and Black women of reproductive age are exposed to SARS-CoV-2 could inform prevention strategies.

Most pregnant women with COVID-19 in this study were asymptomatic, similar to findings in settings where universal SARS-CoV-2 testing is conducted upon admission to labor and delivery units $(\sigma)$. Testing policies based on the presence of symptoms might miss many SARS-CoV-2 infections during

\footnotetext{
$\$$ The distribution of race/ethnicity among the COVID-NET catchment area was calculated using 2019 vintage NCHS bridged-race population estimates. https://www.cdc.gov/nchs/nvss/bridged_race.htm.
}

pregnancy. Early identification of COVID-19 among hospitalized pregnant women can help ensure that health care providers use appropriate personal protective equipment and limit visitors to those essential for patients' well-being and care.

The overall proportion of pregnant women with COVID-19 admitted to an ICU $(7.4 \%)$ was similar to that observed in two European studies $(7,8)$; however, $16.2 \%$ of symptomatic pregnant women in this study were admitted to an ICU, indicating that outcomes might be more severe among pregnant women admitted with acute illness than among those admitted for obstetric indications alone.

Although the preterm delivery rate in the study catchment area during the surveillance period is unknown, the prevalence of preterm delivery among live births during COVID-19-associated hospitalizations $(12.6 \%)$ was higher than that observed in the general U.S. population in 2018 (10.0\%) (9). In this study, preterm births occurred approximately three times more frequently in symptomatic pregnant women than in those who were asymptomatic. Preterm newborns might be at increased risk for severe COVID-19 illness, and preventive measures, such as encouraging caretakers to wear a mask and practice hand hygiene, should be emphasized to minimize possible transmission. ${ }^{* *}$

Birth outcomes in this analysis were limited to pregnancies completed during a COVID-19-associated hospitalization. COVID-NET only captured medically attended pregnancy losses and likely underestimates the percentage of pregnancy losses that occur among women with COVID-19. Further prospective data on birth outcomes among women infected during all pregnancy trimesters is needed. CDC is collaborating with state and local health departments to conduct detailed surveillance of pregnant women with COVID-19 and their infants. ${ }^{\dagger \dagger}$

The findings in this report are subject to at least six limitations. First, at the time of analysis, chart abstractions were ongoing and completed for a convenience sample of $29.4 \%$ of women aged 15-49 years. Thus, the estimated proportion of hospitalized women with COVID-19 who were pregnant might be biased, because pregnancy status was not yet ascertained for women without completed chart review. Second, pregnant women included in this analysis might not be representative of all pregnant women within the catchment area. Third, COVID-19 cases might have been missed because of testing practices and test availability, which likely varied across time and facilities. Fourth, the reason for hospital admission was unavailable for $45.8 \%$ of women, limiting the ability to distinguish between admissions solely for labor and delivery

\footnotetext{
https://www.cdc.gov/coronavirus/2019-ncov/hcp/inpatient-obstetrichealthcare-guidance.html.

** https://www.cdc.gov/coronavirus/2019-ncov/hcp/caring-for-newborns.html.

†† https://www.cdc.gov/coronavirus/2019-ncov/cases-updates/specialpopulations/pregnancy-data-on-covid-19.html; https:/www.cdc.gov/ncbddd/ aboutus/pregnancy/emerging-threats.html.
} 


\section{Summary}

What is already known about this topic?

Information on the clinical characteristics and birth outcomes of hospitalized U.S. pregnant women with COVID-19 is limited.

What is added by this report?

Among 598 hospitalized pregnant women with COVID-19, 55\% were asymptomatic at admission. Severe illness occurred among symptomatic pregnant women, including intensive care unit admissions (16\%), mechanical ventilation ( $8 \%)$, and death (1\%). Pregnancy losses occurred for $2 \%$ of pregnancies completed during COVID-19-associated hospitalizations and were experienced by both symptomatic and asymptomatic women.

What are the implications for public health practice?

Pregnant women and health care providers should be aware of potential risks for severe COVID-19, including adverse pregnancy outcomes. Identifying COVID-19 during birth hospitalizations is important to guide preventive measures to protect pregnant women, parents, newborns, other patients, and hospital personnel.

and those for COVID-19-related illness. Fifth, information on obesity as an underlying prepregnancy condition was not available, so this underlying health condition could not be described. Finally, information on maternal and newborn mortality was only obtained from the maternal medical chart and did not capture outcomes occurring beyond the COVID-19-associated hospitalization.

Severe illness and adverse birth outcomes were observed among hospitalized pregnant women with COVID-19. These findings highlight the importance of preventing and identifying COVID-19 in pregnant women. Pregnant women should avoid close contact with persons with confirmed or suspected COVID-19, maintain 6 feet of distance from nonhousehold members, and take general COVID-19 preventive measures, including wearing masks and practicing hand hygiene. $\$ \$$ CDC recommends testing newborns born to mothers with COVID-19, isolation of mothers with COVID-19 and their newborns from other hospitalized mothers and newborns, and infection prevention measures for persons caring for newborns who might be exposed to SARS-CoV-2. Continued surveillance for COVID-19 in pregnant women is important to understand and improve health outcomes for mothers and newborns.

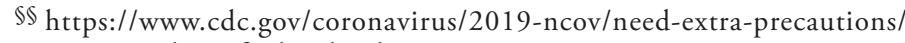
pregnancy-breastfeeding.html.
}

\section{Acknowledgments}

Alexander Flood-Bryzman, Kimberly Gonzalez Barrera, Quynh Ho, Monica Napoles Serrano, California Emerging Infections Program, Oakland, California; Gaggan Brar, Hazal Kayalioglu, Linda Niccolai, Connecticut Emerging Infections Program, Yale School of Public Health, New Haven, Connecticut; Matthew Cartter, Connecticut Department of Public Health; Emily Fawcett, Siyeh Gretzinger, Katelyn Lengacher, Jeremiah Williams, Emerging Infections Program, Georgia Department of Health, Veterans Affairs Medical Center, Foundation for Atlanta Veterans Education and Research; Elisabeth Vaeth, Cindy Zerrlaut, Maryland Department of Health; Jim Collins, Sam Hawkins, Justin Henderson, Shannon Johnson, Libby Reeg, Val Tellez Nunez, Michigan Department of Health and Human Services; Austin Bell, Kayla Bilski, Emma Contestabile, Kristen Ehresmann, Claire Henrichsen, Emily Holodnick, Melissa McMahon, Lisa Nguyen, Katherine Schleiss, Kirk Smith, Minnesota Department of Health; Adam Rowe, New York State Department of Health; Sophrena Bushey, Maria Gaitan, RaeAnne Kurtz, Savanah Russ, University of Rochester School of Medicine and Dentistry, New York; Kathy Billings, Katie Dyer, Anise Elie, Karen Leib, Terri McMinn, Danielle Ndi, Manideepthi Pemmaraju, John Ujwok, Vanderbilt University Medical Center, Nashville, Tennessee; Mary Hill, Ilene Risk, Salt Lake County Health Department, Salt Lake City, Utah; Keegan McCaffery, Utah Department of Health; Mimi Huynh, Monica Schroeder, Council of State and Territorial Epidemiologists, Atlanta, Georgia; Rainy Henry, Sonja Mali Nti-Berko, Robert W. Pinner, CDC.

\section{COVID-NET Surveillance Team}

Mirasol Apostol, California Emerging Infections Program; Susan Brooks, California Emerging Infections Program; Ashley Coates, California Emerging Infections Program; Linda Frank, California Emerging Infections Program; Brooke Heidenga, California Emerging Infections Program; Kareena Hundal, California Emerging Infections Program; Joelle Nadle, California Emerging Infections Program; Sherry Quach, California Emerging Infections Program; Jeremy Roland, California Emerging Infections Program; Maria Rosales, California Emerging Infections Program; Isaac Armistead, Colorado Department of Public Health and Environment; Rachel Herlihy, Colorado Department of Public Health and Environment; Sarah McLafferty, Colorado Department of Public Health and Environment; Adam Misiorski, Connecticut Emerging Infections Program, Yale School of Public Health; Christina Parisi, Connecticut Emerging Infections Program, Yale School of Public Health; Danyel Olson, Connecticut Emerging Infections Program, Yale School of Public Health; Carol Lyons, Connecticut Emerging Infections Program, Yale School of Public Health; Amber Maslar, Connecticut Emerging Infections Program, Yale School of Public Health; Paula Clogher, Connecticut Emerging Infections Program, Yale School of Public Health; David Blythe, Maryland Department of Health; Alicia Brooks, Maryland Department of Health; Rachel Park, Maryland 
Emerging Infections Program, Johns Hopkins Bloomberg School of Public Health; Michelle Wilson, Maryland Emerging Infections Program, Johns Hopkins Bloomberg School of Public Health; Erica Bye, Minnesota Department of Health; Kathryn Como-Sabetti, Minnesota Department of Health; Richard Danila, Minnesota Department of Health; Maureen Sullivan, Minnesota Department of Health; Kathy M. Angeles, New Mexico Emerging Infections Program; Melissa Christian, New Mexico Emerging Infections Program; Nancy Eisenberg, New Mexico Emerging Infections Program; Caroline Habrun, New Mexico Emerging Infections Program; Emily B. Hancock, New Mexico Emerging Infections Program; Sarah A. Khanlian, New Mexico Emerging Infections Program; Meaghan Novi, New Mexico Emerging Infections Program; Yadira Salazar-Sanchez, New Mexico Emerging Infections Program; Elizabeth Dufort, New York State Department of Health; Nancy Spina, New York State Department of Health; Ama OwusuDommey, Public Health Division, Oregon Health Authority; Tiffanie Markus, Vanderbilt University Medical Center; Ryan Chatelain, Salt Lake County Health Department; Laine McCullough, Salt Lake County Health Department; Jake Ortega, Salt Lake County Health Department; Andrea Price, Salt Lake County Health Department; Ashley Swain, Salt Lake County Health Department; Anita Kambhampati, CDC COVID-NET Team, Cherokee Nation Assurance; Seth Meador, CDC COVID-NET Team.

Corresponding author: Miranda J. Delahoy; MDelahoy@cdc.gov.

${ }^{1}$ CDC COVID-NET Team; ${ }^{2}$ Epidemic Intelligence Service, CDC; ${ }^{3}$ Eagle Global Scientific, Atlanta, Georgia; ${ }^{4}$ California Emerging Infections Program, Oakland California; ${ }^{5}$ Colorado Department of Public Health and Environment; ${ }^{6}$ Connecticut Emerging Infections Program, Yale School of Public Health, New Haven, Connecticut; ${ }^{7}$ Departments of Pediatrics and Medicine, Emory University School of Medicine, Atlanta, Georgia; ${ }^{8}$ Emerging Infections Program, Atlanta Veterans Affairs Medical Center, Atlanta, Georgia; ${ }^{9}$ Foundation for Atlanta Veterans Education and Research, Decatur, Georgia; ${ }^{10}$ Maryland Department of Health; ${ }^{11}$ Michigan Department of Health and Human Services; ${ }^{12}$ Minnesota Department of Health; ${ }^{13}$ New Mexico Emerging Infections Program, Albuquerque, New Mexico; ${ }^{14} \mathrm{New}$ Mexico Department of Health; ${ }^{15} \mathrm{New}$ York State Department of Health; ${ }^{16}$ University of Rochester School of Medicine and Dentistry, Rochester, New York; ${ }^{17}$ Ohio Department of Health; ${ }^{18}$ Public Health Division, Oregon Health Authority, Portland, Oregon; ${ }^{19}$ Vanderbilt University Medical Center, Nashville, Tennessee; ${ }^{20}$ Salt Lake County Health Department, Salt Lake City, Utah; ${ }^{21}$ Cherokee Nation Assurance, Arlington, Virginia.
All authors have completed and submitted the International Committee of Medical Journal Editors form for disclosure of potential conflicts of interest. Evan J. Anderson reports grants from MedImmune, Regeneron, PaxVax, Pfizer, GSK, Merck, Novavax, Sanofi-Pasteur, Micron, and Janssen, and personal fees from AbbVie, Pfizer, Sanofi Pasteur and Kentucky BioProcessing, Inc. outside the submitted work. William Schaffner reports personal fees from VBI Vaccines, outside the submitted work. No other potential conflicts of interest were disclosed.

\section{References}

1. Ellington S, Strid P, Tong VT, et al. Characteristics of women of reproductive age with laboratory-confirmed SARS-CoV-2 infection by pregnancy status-United States, January 22-June 7, 2020. MMWR Morb Mortal Wkly Rep 2020;69:769-75. https://doi.org/10.15585/ mmwr.mm6925a1

2. Panagiotakopoulos L, Myers TR, Gee J, et al. SARS-CoV-2 infection among hospitalized pregnant women: reasons for admission and pregnancy characteristics-eight U.S. health care centers, March 1-May 30, 2020. MMWR Morb Mortal Wkly Rep 2020. Epub September 16, 2020.

3. Garg S, Kim L, Whitaker M, et al. Hospitalization rates and characteristics of patients hospitalized with laboratory-confirmed coronavirus disease 2019-COVID-NET, 14 states, March 1-30, 2020. MMWR Morb Mortal Wkly Rep 2020;69:458-64. https://doi.org/10.15585/mmwr. mm6915e3

4. Martinez DA, Hinson JS, Klein EY, et al. SARS-CoV-2 positivity rate for Latinos in the Baltimore-Washington, DC region. JAMA 2020;324:392-5. https://doi.org/10.1001/jama.2020.11374

5. Okoh AK, Sossou C, Dangayach NS, et al. Coronavirus disease 19 in minority populations of Newark, New Jersey. Int J Equity Health 2020;19:93. https://doi.org/10.1186/s12939-020-01208-1

6. Pettirosso E, Giles M, Cole S, Rees M. COVID-19 and pregnancy: a review of clinical characteristics, obstetric outcomes and vertical transmission. Aust N Z J Obstet Gynaecol 2020;ajo.13204. https://doi. org/10.1111/ajo.13204

7. Badr DA, Mattern J, Carlin A, et al. Are clinical outcomes worse for pregnant women at $\geq 20$ weeks' gestation infected with COVID-19? A multicenter case-control study with propensity score matching. Am J Obstet Gynecol 2020. https://doi.org/10.1016/j.ajog.2020.07.045

8. Savasi VM, Parisi F, Patanè L, et al. Clinical findings and disease severity in hospitalized pregnant women with coronavirus disease 2019 (COVID-19). Obstet Gynecol 2020;136:252-8. https://doi.org/10.1097/ AOG.0000000000003979

9. Martin JA, Hamilton BE, Osterman MJK, Driscoll AK. Births: final data for 2018. Natl Vital Stat Rep 2019;68:1-47. 